\title{
Contributions and Heritage of Hector Rubinstein History of the Constituent Quark Model New Challenges for QCD
}

\author{
Harry J. Lipkin** \\ Department of Particle Physics, Weizmann Institute of Science, Rehovot, Israel and \\ High Energy Physics Division, Argonne National Laboratory, Argonne, IL 60439-4815, USA \\ E-mail: ftlipkin@weizmann.ac.il
}

In 1966 Hector Rubinstein came to the Weizmann Institute and worked on the quark model. Then nearly all particle physicists believed that nucleons and pions were elementary, like electrons and photons and that quarks were nonsense. Hector believed that quarks were real, even though we did not know what they were. He then used the quark structure for hadrons to make predictions testable by experiment. He first joined Lipkin and Scheck on quark model predictions. He then directed a student Hannah Stern, later Stern-Kluborg to investigate proton-antiproton annihilation using a revolutionary and controversial model in which the initial three quarks and three antiquarks rearranged to create three mesons. This was the only model that fit the experimental data and showed conclusively for the first time that a nucleon was made of three quarks. But the particle physics establishment rejected this work because they knew that quarks were nonsense. Hector continued with further work on the quark model and then turned to other areas. Now Hector has left us much too soon, but his quark model predictions still influence new experimental analyses

This research was supported in part by the U.S. Department of Energy, Division of High Energy Physics, Contract DE-AC02-06CH11357.

Quarks, Strings and the Cosmos - H??ctor Rubinstein Memorial Symposium August 09-11, 2010

AlbaNova )Stockholm) Sweden

\footnotetext{
${ }^{*}$ Speaker.

${ }^{\dagger}$ A footnote may follow.
} 


\section{A Historical Perspective}

\subsection{The conflict between "Grand Unification" and "Compositeness"}

Each stage in the development of our knowledge of the structure of matter began with "known" fundamental constituents of matter or "elements". Experimental discoveries of too many elements led first to unifying elements while still considered them elementary. Chemical elements were unified in the Mendeleev Periodic Table. The large number of particles was unified by symmetry classifications. Then each element was shown to be built from smaller fundamental building blocks, as in the Bohr model of atom and the quark model of hadrons.

In 1950 the $N$ and $\pi$ were considered fundamental constituents of hadronic matter. Attempts were made to unify all the new "elementary" particles as equally elementary, using concepts like nuclear democracy or higher symmetry. Evidence for composite structure was resisted by the establishment.

\subsection{SU(3) symmetry - the Mendeleev table for elementary particles}

In 1960 Murray Gell-Mann and Yuval Ne'eman introduced SU3 octet classifications for the known mesons and baryons. Particles with the same spin and baryon number were grouped into multiplets and classified into octet representations of the group SU(3) containing two isospin doublets with hypercharge +1 and -1 and an isospin triplet an an isospin singlet with hypercharge zero.

Later it was noticed that the same particles could also be classified in " barbaryon" octets. Particles with the same isospin and hypercharge were grouped into octets containing two spin doublets with baryon number +1 and -1 and a spin triplet and a spin singlet with baryon number zero. These two $S U(3)$ groups could then be combined into a group $S U(3) \otimes S U(3)$ having an $(8,8)$ representation containing all the 64 known mesons, baryons and antibaryons.

No reasons were given for the number 3 in SU(3) and why all particles were grouped into the octet representations of SU(3). Grouping both fermions and bosons in the same multiplets anticipated what is now called supersymmetry but had no physical basis at that time.

\section{The Meson Octet}

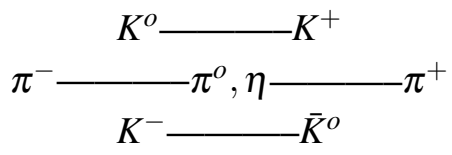

The Baryon Octet
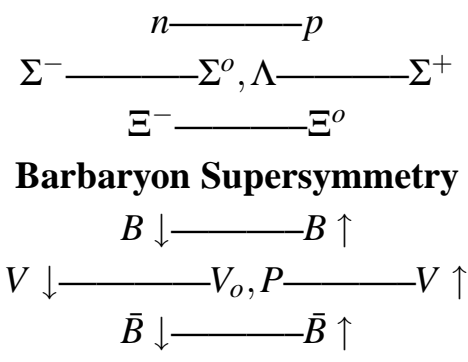

\subsection{The journey from nucleons and pions to quarks and gluons}

1. The journey began by noting that there were too many hadrons 
2. Gell-Mann and Ne'eman classified the nucleon and pion into SU(3) octets

3. No reason for the number 3 in $\mathrm{SU}(3)$; No reason for octets

4. No reason why mesons and baryons should both be in octet

5. Mesons and baryons were very different, like electron and photon

The experimental discovery of new particles that could not be grouped into octets created a crisis. The $\Delta$ with spin (3/2) and isospin (3/2) fell out of both octet classifications. There were also the $\eta$ and $\phi$ mesons which did not fit in anywhere. Okubo showed that these new mesons could be grouped into nonets of U(3), rather than SU(3). Each nonet contained an SU(3) singlet and an SU(3) octet. The SU(3) singlet and octet states in a U(3) multiplet having the same iospin and hypercharge could mix in forming the physical particles. This was particularly evident in the vector mesons. The nine states in the nonet should be classified in the $S U(2) \otimes U(1)$ subgroup of $U(3)$ rather than the $S U(3)$ subgroup.

But the $\Delta$ could not fit into an octet. This required finding new representations of SU(3) which could include a particle with isospin (3/2). It was classified in a decuplet of SU(3) by Glashow and Sakurai who then predicted the existence of the particle now called the $\Omega^{-}$. The consensus that symmetry made sense was finally established when the $\Omega^{-}$was found at Brookhaven. But this killed the barbaryon classification which could not include a state with isospin (3/2) and spin (3/2).

The explanations of too many elementary objects now moved from the nucleon-pion level to the quark-gluon level. Fundamental triplets were introduced by Goldberg, Ne'eman, Gell-Mann and Zweig. That no $K^{+} N$ resonances were found was called the "Goldhaber Gap" by Gell-Mann and led to quarks. The absence of exotic hadrons made from more than three basic bilding blocks was an early clue to QCD

The quark model led to understanding hadron structure in many ways. The simple additive quark model (AQM) 11,2$]$ of Levin and Frankfurt explained the ratio of $3 / 2$ between $\sigma_{\text {tot }}(N N)$ and $\sigma_{t o t}(\pi N)$ This showed that mesons and baryons were not elementary but made of the same quarks.

\section{The Pre-History of QCD}

\subsection{The Sakharov Zeldovich quark model}

Andrei Sakharov took quarks seriously in 1966. He asked "The $\Lambda$ and $\Sigma$ are made of same quarks. Why are masses different?" Sakharov and Zeldovich anticipated QCD by showing that mesons and baryons were made of the same quarks. Their quark model had a mass operator with a flavor dependent linear term for the "effective" quark masses and a flavor-dependent hyperfine interaction.

$$
v_{i j}=v_{i j}^{o}+\vec{\sigma}_{i} \cdot \vec{\sigma}_{j} v_{i j}^{h y p}
$$

They obtained two surprising meson - baryon mass relations

$$
\begin{aligned}
& \left(m_{s}-m_{u}\right)_{B a r}=M_{\Lambda}-M_{N}=177 \mathrm{MeV} ; \quad\left(m_{s}-m_{u}\right)_{M e s}=\frac{\left.3\left(M_{K^{*}}-M_{\rho}\right)+M_{K}-M_{\pi}\right)}{4}=180 \mathrm{MeV} \\
& 1.53=\frac{M_{\Delta}-M_{N}}{M_{\Sigma^{*}}-M_{\Sigma}}=\frac{M_{\rho}-M_{\pi}}{M_{K^{*}}-M_{K}}=1.61
\end{aligned}
$$




\subsection{Static hadron properties with quarks}

\section{The Very Early Successes}

1. Explained difference between the quark structures of the meson and baryon octets

2. Explained striking regularities beyond $\mathrm{SU}(3)$ in low-lying hadron spectrum

3. Baryon octets and decuplets; meson nonets and no meson decuplets

4. No ninth baryon suggested by some SU(3) models

5. Spin-parity quantum numbers $J^{P}=0^{-}, 1^{-}, 1 / 2^{+}, 3 / 2^{+}$.

Introduceing $\mathrm{U}(3)$ rather than $\mathrm{SU}(3)$, breaking $\mathrm{SU}(3)$ at quark level and setting $m_{s}>m_{u} \mathrm{im}$ mediately gave experimentally observed mass inequalities

$M_{\Xi}>M_{\Sigma} \approx M_{\Lambda}>M_{N} ; \quad M_{\eta}>M_{K^{+}} \approx M_{K^{-}}>M_{\pi}$

\subsection{Many open questions remained}

1. Spin and statistics

2. Reason for decuplet classification for the spin-3/2 baryons

3. Reason for the $\Lambda-\Sigma$ mass difference

4. Were next excited states orbital excitations or more $\bar{q} q$ pairs

\subsection{Hadron reactions in the quark model; Further evidence for a quark structure of hadrons}

1. The additive quark model for hadron reactions

2. The so-called ideal mixing pattern of vector and tensor mesons,

3. Peculiar systematics in energy behavior of certain hadron $\sigma_{t o t}$.

4. Mysterious topological quark diagram selection rule now called OZI

- Neutral Meson Mixing and OZI

- First use of the additive quark model to obtain OZI relations

- Selection rule forbidding reactions like $\sigma\left(\pi^{-} p \rightarrow N \phi\right)=0$

- Its $\mathrm{SU}(3)$ rotation predicting the equality $\sigma\left(K^{-} p \rightarrow \Lambda \omega\right)=\sigma\left(K^{-} p \rightarrow \Lambda \rho^{o}\right)$

- The $\rho^{o}$ and $\omega$ mesons produced equally.only via their $u \bar{u}$ component 


\subsection{Nambu universal meson - baryon mass formula (1966)}

Nambu solved the exotics problem in 1966 !

Nambu's model with colored quarks and non-abelian SU(3) gauge field predicted that only hadrons with constituents $(q q q)$ and $(\bar{q} q)$ would be stable. There would be no "exotic states with more constituents.

Nambu's Theorem: Only lowest color singlets are stable if the quark level interaction was described by a color-exchange two-body potential

$V_{c x}\left(r_{i j}\right)=V \cdot \lambda_{c}^{i} \cdot \lambda_{c}^{j} \cdot v\left(r_{i j}\right) ; \quad \vec{\lambda}_{c}$ color SU(3) generator

For color singlet states having $\mathrm{N}$ quarks and antiquarks

$\left\langle V_{c x}(\right.$ tot $\left.)\right\rangle=\sum_{i \neq j} \frac{V \lambda_{c}^{i} \cdot \lambda_{c}^{j}}{2} \cdot\langle v(r)\rangle=\frac{V}{2} \cdot\left[\sum_{i}\left(\lambda_{c}^{i}\right)^{2}\right] \cdot\langle v(r)\rangle=\frac{N V}{2}\left(\lambda_{c}^{i}\right)^{2} \cdot\langle v(r)\rangle$

In lowest order neglecting color-space and color-spin correlations This descripion gave surprising agreement with experiment.

\section{The beginning of Hector at the Weizmann Institute}

\subsection{Hector's arrival and work on quarks}

At the time in 1966 when Hector arrived at the Weizmann Institute nearly all particle physicists elsewhere believed that nucleons and pions were elementary, like electrons and photons. Nucleons coupled to pions like electrons coupled to photons. The pion exchange force bound nucleons into nuclei like photon exchange bound electrons and nuclei into atoms. But the high energy theory group at the Weizmann Institute no longer believed that nucleons were elementary and worked on the quark model. Although the majority of particle physicists at that time were sure that quarks were nonsense, the Weizmann group had already found[1, 2] that the quark model explained experimental data that the accepted model could not. Hector immediately joined this work and collaborated on a number of papers $[3,4,5,6]$.

\subsection{First original application of the quark model $\bar{p} p$ annihilation at rest}

\subsubsection{Before Hector}

The standard description of nucleon-antinucleon annihilation into pions was believed to be like electron-positron annihilation into photons. The baryon numbers of the nucleon and antinucleon were annihilated and pions were created from the vacuum. The statistical models in common use with elementary nucleons and pions predicted the number of pions creation from the vacuum by analogy with the number of photons created in electron-positron annihilation. The pion multiplicity of 2 or 3 predicted by these statistical models disagreed strongly with the experiments which showed a much larger pion multiplicity of $5.3 \pm 0.4$. No $e^{+}-e^{-}$pairs were seen at level predicted by QED for the creation from the vacuum.

\subsubsection{Enter Hector Rubinstein - With typical Hector enthusiasm}

Quarks are real! How do we look for them? Nucleons and pions were not elementary, they were made of quarks with baryon number (1/3). In $\bar{p} p$ annihilation no baryon number needs to 
be annihilated and no mesons need to be created from the vacuum. The quarks and antiquarks are already there. The three quarks and three antiquarks in the nucleon-antinucleon system rearranged themselves into three mesons. Hector's unacceptable heretical explanation was that mesons and baryons were composite. Rearrangement of $3 q$ and $3 \bar{q}$ in $p \bar{p}$ into 3 mesons predicted final state pion multiplicity 5.25. This agreed with the observed experimental value $5.3 \pm 0.4 !$ ! ! Hector's rearrangement model fit the data, but the establishment knew it was nonsense. The quarkrearrangement model was ridiculed when Hector proposed it in 1966.

\subsubsection{The establishment prejudice against quarks created serious problems}

- There were difficulties for obtaining appointment and promotion for Hector

- Deans and committees were influenced by letters from well-known physicists

- All condemned people who rush into print with such quark garbage.

\subsection{Further refinements by Hector successfully described many other experiments}

The universality of additive quark couplings to mesons and baryons arose again and again in different contexts. An S-matrix Regge approach beginning with finite-energy sum rules led to the duality revolution and the Veneziano model.

1. Same states appear as s-channel resonances and t-channel exchanges.

2. Dual resonance models beginning with the Veneziano model.

3. Quark-model constraints on Reggeon couplings provided powerful input with predictive power.

4. Absence of exotics as resonances and t-channel exchanges led to OZI.

Exchange degeneracy and $\bar{q} q$ annihilation dominance of of $\sigma_{t o t}$ led naturally to duality diagrams.

The energy constant part of cross section, later found to be slowly rising, was related to diffraction, described by Pomeron exchange with coupling given by the Levin-Frankfurt quark-counting recipe.

\subsection{Hector's later heritage includes further work on the quark model}

One example is Electromagnetic production of Tensor Mesons. In 2009 HERA looked for resonances and glueballs in ep collisions[7]. They looked for states decaying into two neutral kaons, fit the known states by Breit-Wigner curves and looked for more states. The Breit-Wigner model did not fit the data on the $f_{o}, A_{2}$ and $f^{\prime}$ tensor mesons. The 1975 paper by Hector et al [8] showed them how to analyze their data.

\subsection{The November revolution and heavy quarks}

\subsubsection{Charmonium, the hydrogen atom of particle physics}

Successful description as a heavy quark-antiquark pair interacting via a nonrelativistic potential. But the flavor dependence of the relation between mesons and was baryons not understood 


\subsubsection{The present challenge to QCD - Same as faced by Hector - at a deeper level}

We can use the fact that QCD somehow makes hadrons from quarks and gluons. But we don't understand why this succeeds.

The constituent quark model uses QCD phenomenology now with five flavors and a successful nuclear physics approach. This leads to surprising agreement with experiment. The observation that all nuclei were built of the same neutrons and protons was extended led to the assumption that all known hadrons including $N, \pi, \rho, \Delta, \Lambda, \Sigma, \Sigma^{*}$,etc were built from the same constituent $(\mathrm{u}, \mathrm{d}, \mathrm{s})$ quarks

But we still don't understand why it succeeds. QCD theoretical treatments have not yet been able to explain why this constituent quark model gives surprising relations between mesons and baryons in agreement with experiment[9].

\section{References}

[1] E.M. Levin and L.L. Frankfurt, JETP Lett.2:65,1965.

[2] H.J. Lipkin and F. Scheck, Phys.Rev.Lett.16:71,1966.

[3] H.R. Rubinstein, Phys.Rev.Lett.17, 41, (1966)

[4] H.R. Rubinstein, F. Scheck, and R.H. Socolow, Phys.Rev.154:1608 (1967).

[5] H.R. Rubinstein and H. Stern, Phys. Lett. 21 (1966) 447 Dynamical Derivation Of Baryon Masses In The Quark Model.

[6] P. Federman, H.R. Rubinstein and I. Talmi, Phys. Lett. 22 ( 1966) 208

[7] ZEUS collaboration, S. Chekanov er al Phys.Rev.Lett.101, 112003 2008)

[8] D. Faiman, H.J. Lipkin and H.R. Rubinstein, Phys.Lett.B59:269,1975.

[9] Marek Karliner, Boaz Keren-Zur, Harry J. Lipkin and Jonathan L. Rosner, Annals Phys.324:2,(2009) 